\title{
OBESIDADE E RISCO CORONARIANO EM HOMENS E MULHERES PARTICIPANTES DE UM PROJETO DE EXTENSÃO UNIVERSITÁRIA
}

Elaine Aparecida de Oliveira, Paula Rapchan dos Santos Torquato, Thais Cabral Almeida, Vitor Eduardo dos Santos Silva, Rafaella de Sá Turini Alves, Maria Isabela Ramos Haddad, Gisele Carla Gonçalves dos Santos, Laís Manata Vanzella, Anne Kastelianne França da Silva, Luiz Carlos Marques Vanderlei

Universidade Estadual Paulista - UNESP, Curso de Fisioterapia, Presidente Prudente, SP. e-mail: elaineoloiveira76@gmail.com

\section{RESUMO}

Caracterizada pelo acúmulo excessivo de gordura corporal a obesidade se torna um problema de saúde pública com o avanço da idade podendo ser avaliada por meio do índice de massa corpórea (IMC) e o Índice de conicidade (IC). O objetivo foi determinar a prevalência de obesidade e comparar risco coronariano em homens e mulheres participantes do programa de prevenção primária e secundária de doenças cardiovasculares de um centro de reabilitação. Os voluntários foram identificados e tiveram avaliadas medidas antropométricas e de circunferência da cintura para o cálculo do IMC e IC. O método estatístico utilizado foi Shapiro-Wilk para normalidade e posterior teste $t$ de Student e Man-Withney $(p<0,05)$. Foi observado predomínio de sobrepeso, e maior risco coronariano no sexo masculino, com diferenças significantes neste último. Concluiu-se que há prevalência de sobrepeso na população avaliada e maior risco coronariano em homens, sendo indicados programas preventivos que podem amenizar este fator prevenindo eventos cardiovasculares.

Palavras-chave: obesidade, risco coronariano, doenças cardiovasculares, prevenção, fatores de risco.

\section{OBESITY AND CORONARY RISK IN MEN AND WOMEN OF PARTICIPANTS A UNIVERSITY EXTENSION PROJECT}

\begin{abstract}
Characterized by excessive accumulation of body fat obesity becomes a public health problem with increasing age can be assessed using the body mass index (BMI) and conicity index (CI). The objective was to determine the prevalence of obesity and compare coronary risk in men and women participating in the primary prevention program and secondary of cardiovascular disease of rehabilitation center. The volunteers were identified and were evaluated anthropometric measures and waist circumference for the calculation of $\mathrm{BMI}$ and $\mathrm{Cl}$. The statistical method used was Shapiro-Wilk for normality and later Student's $\mathrm{t}$ test and Man-Whitney $(\mathrm{p}<0.05)$. I was observed the prevalence of overweight it is, and major coronary risk in males, with significant differences in the latter. It was concluded the prevalence of overweight evaluated and increased coronary risk in men population, being indicated prevention programs that can mitigate this factor preventing cardiovascular events.
\end{abstract}

Keywords: obesity, coronary risk, cardiovascular diseases, prevention, risk factors

\section{INTRODUCÃO}

A obesidade é caracterizada pelo acúmulo excessivo de gordura corporal na forma de tecido adiposo que desencadeiam prejuízos na saúde da população, estando relacionada à fatores hereditários, alterações hormonais e fatores ambientais ou sociais, ao qual pode-se citar a má alimentação e o estresse ${ }^{1,2}$.
Com o avanço da idade a gordura é facilmente adquirida e difícil de ser eliminada, tornando a obesidade um problema de saúde pública de importância ainda maior ${ }^{3}$. Além disso, sua prevalência vem aumentando ao longo dos anos juntamente com número de mortes por doenças relacionadas ao excesso de peso ${ }^{4,5}$.

Segundo dados da Organização Mundial da Saúde (OMS), o sobrepeso atinge cerca de 1,6 
bilhões de indivíduos acima de 15 anos, sendo considerados obesos cerca de 400 milhões destes $^{6}$. No período de 2006 a 2012, a prevalência da obesidade na população adulta das capitais dos 26 estados brasileiros e o Distrito Federal aumentou de $11,6 \%$ para $17,4 \%$, o que representa um incremento médio de $0,89 \%$ ao ano?.

A obesidade, principalmente a abdominal, predispõe ao aparecimento de diversos fatores de risco cardiovasculares como as dislipidemias, hipertensão arterial, resistência à insulina e diabetes que favorecem a ocorrência de eventos cardíacos, especialmente aqueles de origem coronariana $^{8,9}$.

Diversos são os indicadores de obesidade, podendo ser destacados o Índice de Massa Corporal (IMC) e o índice de Conicidade (IC). O IMC apesar de muito utilizado não se correlaciona totalmente à distribuição da gordura corporal $^{10}$, porém é recomendado pela Organização Mundial da Saúde (OMS) e considerado padrão outro para identificação de pacientes com risco de saúde havendo associação direta com o risco de complicações médicas e taxa de mortalidade ${ }^{11}$. Já o IC é determinado por medidas do peso, altura e circunferência da cintura e vem demonstrando forte correlação com os fatores de risco cardiovascular ${ }^{9,12}$ podendo ser utilizado inclusive para discriminar alto risco coronariano ${ }^{13}$.

Neste estudo, o objetivo foi determinar a prevalência de obesidade e comparar risco coronariano em homens e mulheres participantes de um programa de prevenção primária e secundária de doenças cardiovasculares de um centro de reabilitação.

\section{MATERIAL E MÉTODOS}

Participaram do estudo 25 indivíduos, sendo 14 homens com idade de $68,28 \pm 12,25$ anos e 11 mulheres com idade de $67,18 \pm 11,89$ anos, todos participantes de um projeto de prevenção em um centro de reabilitação. Os procedimentos do estudo foram aprovados pelo Comitê de Ética em Pesquisa da FCT/UNESP (CAAE: 17442413.0.0000.5402).
Os voluntários foram previamente orientados a portarem roupas leves na data da avaliação. Os mesmos foram identificados quanto ao nome, idade e sexo, e tiveram avaliadas as medidas antropométricas (massa corporal e altura) e medidas de circunferência da cintura (CC).

A massa corporal foi obtida por meio de uma balança digital (Maxima, Brasil) e a medida de altura realizada por meio de um estadiômetro (Sanny, Brasil) com os voluntários em posição ortostática, com os pés paralelos e sem sapatos, o peso do corpo distribuído de maneira uniforme entre eles e permanecendo de costas durante a medida. Já para obtenção da CC foi utilizado uma fita métrica (Sanny, Brasil) posicionada na região mais estreita do abdome. A partir desses dados, foi calculado o IMC através da relação peso/altura ${ }^{2}\left(\mathrm{~kg} / \mathrm{m}^{2}\right)$, para determinação e classificação de obesidade, conforme as Diretrizes Brasileiras de Obesidade $^{5}$, e o IC calculado utilizando-se a seguinte equação matemática:

$\mathrm{CC}(\mathrm{m}) / 0,109 \mathrm{Vpeso}(\mathrm{kg}) /$ estatura $(\mathrm{m})^{13}$.

Para caracterização da amostra foi utilizado estatística descritiva e os resultados foram apresentados com valores de média, desvio padrão e intervalo de confiança. Para comparação as variáveis entre homens e mulheres, inicialmente foi testada a normalidade dos dados pelo teste de Shapiro-Wilk e posteriormente foi utilizado teste $t$ de Student para dados não pareados ou teste de MannWhitney, com nível de significância de $5 \%$.

\section{RESULTADOS}

$\mathrm{Na}$ Tabela 1 são apresentadas as características dos voluntários dos dois grupos estudados, juntamente com valores de IMC e IC. Maiores valores das variáveis peso, altura, circunferência da cintura e IC foram encontradas nos homens em comparação com as mulheres. Não foram encontradas diferenças estatisticamente significantes entre os grupos para as variáveis Idade e IMC. 
Tabela 1. Caracterização das variáveis antropométricas e índice de conicidade dos voluntários separados por grupo.

\begin{tabular}{lccc}
\hline & Homens & Mulheres & p \\
\hline Idade (anos) & $68,28 \pm 12,25$ & $67,18 \pm 11,89$ & 0,82 \\
& {$[61,21-75,36]$} & {$[59,16-75,16]$} & \\
\hline Peso $(\mathrm{Kg})$ & $76,83 \pm 12,75$ & $65,41 \pm 12,54$ & 0,03 \\
& {$[69,47-84,20]$} & {$[56,99-73,84]$} & \\
\hline Altura (m) & $1,68 \pm 0,06$ & $1,55 \pm 0,08$ & 0,001 \\
& {$[1,64-1,71]$} & {$[1,49-1,60]$} & \\
\hline CC $(\mathrm{cm})$ & $0,95 \pm 0,10$ & $0,85 \pm 0,10$ & 0,02 \\
& {$[0,89-1,02]$} & {$[0,78-0,93]$} & \\
\hline IMC $\left(\mathrm{kg} / \mathrm{m}^{2}\right)$ & $27,08 \pm 3,61$ & $27,22 \pm 4,79$ & 0,80 \\
& {$[24,99-29,16]$} & {$[24,00-30,44]$} & \\
\hline IC & $1,30 \pm 0,08$ & $1,22 \pm 0,11$ & 0,026 \\
& {$[1,26-1,35]$} & {$[1,14-1,30]$} & \\
\hline
\end{tabular}

Média \pm desvio padrão [intervalo de confiança], IMC - índice de massa corpórea;

CC - circunferência da cintura; IC - índice de conicidade.

\section{DISCUSSÃo}

Os resultados apontam que os indivíduos avaliados foram classificados com sobrepeso de acordo com o IMC, porém não houve diferenças significativas quando comparado homens e mulheres. Já com relação ao IC, foram encontradas diferenças estatisticamente significantes entre os grupos avaliados.

Com relação ao IMC o fato de não haver diferenças significativas entre os sexos pode ser justificado por ambos os grupos serem classificados no mesmo estágio através desta variável (sobrepeso). Porém, foi possível observar que o sexo masculino apresenta maiores valores de peso e altura quando comparado com o sexo feminino, assim como encontrado por Pitanga e Lessa $^{12,14}$

Já com relação ao IC, este leva em conta além do peso e altura, os valores de circunferência da cintura, que foram significativamente diferentes entre homens e mulheres, caracterizando os homens com maior acúmulo de gordura na região abdominal, e maior risco coronariano segundo cálculos deste índice.

A distribuição da gordura no corpo tem despertado atenção nas pesquisas sobre obesidade ${ }^{15}$. Apesar de vários índices antropométricos terem sido propostos o IMC talvez seja o de maior divulgação no que diz respeito à mensagem populacional, sendo seus valores limites conhecidos por especialistas e leigos ${ }^{10}$. Porém, segundo Rossi et al. ${ }^{16}$ não pode ser significativamente associado à maior extensão da aterosclerose coronariana, nem à mortalidade.

Com relação ao IC, apesar de existirem algumas discussões sobre o assunto, este é reconhecido como um bom indicador de obesidade central $^{17}$ apresentando poder satisfatório em estudo realizado por Almeida et al. ${ }^{6}$ para discriminar risco coronariano elevado (RCE) obtendo o melhor desempenho quando comparado com os demais índices.

Segundo dados obtidos por Barreto et al. ${ }^{18}$ a probabilidade de desenvolver doença arterial coronariana em 10 anos entre os homens cresceu gradativamente com a faixa etária, atingindo seu pico $(25,1 \%)$ na faixa etária mais alta (70-74 anos), já entre as mulheres, este fenômeno também aconteceu, porém o risco médio mais elevado (13\%) foi pouco mais que a metade do observado para os homens. O maior risco da doença arterial coronariana com o aumento da idade ocorreu devido a uma combinação de fatores, as explicações para essa diferença se relacionam a aspectos biológicos (sexo), culturais e estilo de vida (gênero) ${ }^{18}$.

No sentido de evitar o aparecimento e a combinação destes fatores, programas preventivos contribuem para população com informações capazes de mobilizá-la, facilitar o acesso à informação, identificar indivíduos com maus hábitos de vida e estimular a confirmação diagnóstica de fatores como a hipertensão arterial $^{9}$. Ainda, podem contribuir para a redução do colesterol, pressão arterial sistólica, uso de medicamentos, diminuição do hábito de fumar, e na redução da probabilidade de ocorrência de qualquer evento cardiovascular, como encontrado por Ciorla et al. ${ }^{19}$ ao observar os efeitos de uma intervenção sobre os principais fatores de risco para doenças cardiovasculares ao longo de 20 anos em 621 eletricitários ${ }^{9}$. 


\section{CONCLUSÃO}

Conclui-se que há condição de sobrepeso na população avaliada e ainda, maior risco coronariano em homens quando comparados ao sexo feminino. Programas preventivos podem contribuir favoravelmente e para o controle dos fatores de risco por meio de mudanças no estilo de vida e são de extrema importância nesta população.

\section{CONFLITO DE INTERESSE}

Os autores declaram não haver qualquer potencial de conflito de interesse que possa interferir na imparcialidade deste trabalho científico.

\section{REFERÊNCIAS}

1. World Health Organization. Obesity: Preventing and Managing the Global Epidemic. Report of a WHO Consultation. Geneva; 2004. WHO Technical Report Series no. 894

2. Silva D, Rego C, Valente A, Faria M, Claudia D, Azevedo L. Avaliação da insatisfação corporal e da autoestima em crianças e adolescentes com diagnóstico de obesidade vs uma comunidade escolar. Rev Port Endocrinol, Diab Metabol. 2009;1:23-31.

3. Burton B. Nutrição Humana. São Paulo: Ed. McGraw Hill do Brasil, 1979.

4. Teixeira CA, Santos JE, Silva GA, Souza EST, Martinez JAB. Prevalência de dispnéia e possíveis mecanismos fisiopatológicos envolvidos em indivíduos com obesidade graus 2 e 3. J Bras Pneumol. 2007;33(1):2835. DOI: http://dx.doi.org/10.1590/S1806$\underline{37132007000100008}$

5. Diretrizes brasileiras de obesidade 2009/2010 / ABESO - Associação Brasileira para o Estudo da Obesidade e da Síndrome Metabólica. 3.ed. Itapevi, SP: AC Farmacêutica, 2009.

6. World Health Organization (WHO). Obesity: preventing and managing the global epidemic of obesity. Geneva: WHO; 2004.

7. Malta DC, Andrade SC, Claro RM, Bernal RTI, Monteiro CA. Evolução anual da prevalência de excesso de peso e obesidade em adultos nas capitais dos 26 estados brasileiros e no Distrito Federal entre 2006 e 2012. Rev Bras Epidemiol. 2014;17(Supl 1): 267-76. DOI: http://dx.doi.org/10.1590/1809-4503201400050021.

8. Kannel WB, Wilson PW, Nam BH, D'Agostino RB. Risk stratification of obesity as a coronary risk factor. Am J Cardiol. 2002;90:697-701. DOI:

http://dx.doi.org/10.1016/S0002-9149(02)02592-4

9. Tonstad S, Hjermann I. A high risk score for coronary heart disease is associated with the metabolic syndrome in 40-year-old men and women. J Cardiovasc Risk. 2003; 10:129-35. DOI: http://dx.doi.org/10.1177/174182670301000208

10. OMS. Obesidade: prevenindo e controlando a epidemia global. Relatório da consultoria da OMS. São Paulo: Roca; 2004.

11. Klein S, Allison DB, Heymsfield SB, Kelley DE, Leibel $\mathrm{RL}$, Nonas $\mathrm{C}$, et al. Waist circumference and cardiometabolic risk: a consensus statement from shaping america's health: association for weight management and obesity prevention; NAASO, the Obesity Society; the American Society for Nutrition; and the American Diabetes Association. Obesity. 2007;15(5):1061-7. DOI:

http://dx.doi.org/10.1038/oby.2007.632

12. Lemos-Santos MGF, Valente JG, Gonçalves-Silva, RMV, Sichieri R. Waist circumference and waist-to-hip ratio as predictors of serum concentration of lipids in Brazilian men. Nutrition. 2004;20:857-62. DOI: http://dx.doi.org/10.1016/j.nut.2004.06.005

13. Pitanga FJG. Sensibilidade e especificidade do índice de conicidade como discriminador do risco coronariano de adultos em Salvador, Brasil. Rev Bras Epidemiol. 2004;7(3):259-69.

DOI: http://dx.doi.org/10.1590/S1415-790X2004000300004 14. Pitanga FJG, Lessa I. Associação entre indicadores antropométricos de obesidade e risco coronariano em adultos na cidade de Salvador, Bahia, Brasil. Rev Bras Epidemiol. 2007;10(2):239-48. DOI: http://dx.doi.org/10.1590/S1415-790X2007000200011

15. Koning L, Merchant AT, Pogue J, Anand SS. Waist circumference and waist-to-hip ratio as predictors of cardiovascular events: meta-regression analysis of prospective studies. Eur Heart J. 2007;28(7):850-6. DOI: http://dx.doi.org/10.1093/eurhearti/ehm026

16. Rossi R, laccarino D, Nuzzo A, Chiurlia E, Bacco L, Venturelli $A$, et al. Influence of body mass index on extent of coronary atherosclerosis and cardiac events in a cohort of patients at risk of coronary artery disease. Nutr Metab Cardiovasc Dis. 2011; 21(2):86-93. DOI: http://dx.doi.org/10.1016/i.numecd.2009.09.001

17. Associação Brasileira para o estudo da obesidade e síndrome metabólica. III Diretrizes Brasileira de Obesidade. 2009/2010.

18. Barreto SM, Passos VMA, Cardoso ARA, Lima-Costa MF. Quantificando o Risco de Doença Coronarianana Comunidade. Projeto Bambuí. Arq Bras Cardiol, 2003; 81(6):549-55.

19. Marks, J.B. Advances in obesity treatment: clinical highlights from the NAASO 2003 Annual Meeting. Clin Diabetes. 2004;22:23-6. http://dx.doi.org/10.2337/diaclin.22.1.23

Recebido para publicação em 20/08/2015

Revisado em 17/09/2015

Aceito em 25/09/2015 